\title{
A Strong Method for Solving Systems of Integro-Differential Equations
}

\author{
Jafar Biazar, Hamideh Ebrahimi \\ Department of Applied Mathematics, Faculty of Mathematical Science, University of Guilan, Rasht, Iran \\ E-mail: biazar@guilan.ac.ir,ebrahimi.hamideh@gmail.com \\ Received June 5, 2011; revised June 28, 2011; accepted July 6, 2011
}

\begin{abstract}
The introduced method in this paper consists of reducing a system of integro-differential equations into a system of algebraic equations, by expanding the unknown functions, as a series in terms of Chebyshev wavelets with unknown coefficients. Extension of Chebyshev wavelets method for solving these systems is the novelty of this paper. Some examples to illustrate the simplicity and the effectiveness of the proposed method have been presented.
\end{abstract}

Keywords: Systems of Integro-Differential Equations, Chebyshev Wavelets Method, Mother Wavelet, Operational Matrix

\section{Introduction}

In recent years, many different orthogonal functions and polynomials have been used to approximate the solution of various functional equations. The main goal of using orthogonal basis is that the equation under study reduces to a system of linear or non-linear algebraic equations. This can be done by truncating series of functions with orthogonal basis for the solution of equations and using the operational matrices. In this paper, Chebyshev wavelets basis, on the interval $[0,1]$, have been considered for solving systems of integro-differential equations. There are some applications of Chebyshev wavelets method in the literature [1-3].

Systems of integro-differential equations arise in mathematical modeling of many phenomena. Some techniques have been used for solving these systems such as, Adomian decomposition method (ADM) [4], He's homotopy perturbation method (HPM) $[5,6]$, variational iteration method (VIM) [7], The Tau method [8,9], differential transform method (DTM ) [10], power series method, rationalized Haar functions method and Galerkin method for linear systems [11-13]. The general form of these systems are considered as follows

1) Systems of Volterra integro-differential equations

$$
\begin{aligned}
& u_{i}^{(m)}(x)=f_{i}(x)+\sum_{k=1}^{m_{1}} F_{i k}\left(x, u_{1}(x), u_{1}^{\prime}(x), \cdots, u_{1}^{(m)}(x), \cdots, u_{n}^{(m)}(x)\right) \\
&+\sum_{j=1}^{m_{2}} \int_{0}^{x} k_{i, j}(x, t) G_{i, j}\left(u_{1}(t), \cdots, u_{1}^{(m)}(t), \cdots, u_{n}^{(m)}(t)\right) \mathrm{d} t, \\
& 0 \leq x \leq 1, \quad i=1,2, \cdots, n,
\end{aligned}
$$

2) Systems of Fredholm integro-differential equations

$$
\begin{aligned}
& u_{i}^{(m)}(x)=f_{i}(x)+\sum_{k=1}^{m_{1}} F_{i k}\left(x, u_{1}(x), u_{1}^{\prime}(x) \cdots, u_{1}^{(m)}(x), \cdots, u_{n}^{(m)}(x)\right) \\
&+\sum_{j=1}^{m_{2}} \int_{0}^{1} k_{i, j}(x, t) G_{i, j}\left(u_{1}(t), \cdots, u_{1}^{(m)}(t), \cdots, u_{n}^{(m)}(t)\right) \mathrm{d} t, \\
& 0 \leq x \leq 1, \quad i=1,2, \cdots, n,
\end{aligned}
$$

where $m, m_{1}$ and $m_{2}$ are positive integers,

$$
k_{i j}(x, t) \in L^{2}([0,1] \times[0,1]) \text { are the kernels, }
$$


$f_{i}(x), i=1,2, \cdots, n$, are known functions, $F_{i k}$ and $G_{i j}$ are linear or non-linear functions and

$u_{i}(x), i=1,2, \cdots, n$, are unknown functions.

This paper is organized as follows: in Section 2, Chebyshev wavelets method is explained. In Section 3, the application of the method for introduced systems (1) and (2) is studied. Some numerical examples are presented in Section 4, Conclusions are presented in Section 5.

\section{Wavelets and Chebyshev Wavelets}

Wavelets constitute a family of functions constructed from dilation and translation of a single function called the mother wavelet $[14,15]$. When the dilation parameter $a$ and the translation parameter $b$ vary continuously we have the following family of continuous wavelets

$$
\psi_{a, b}(x)=|a|^{-\frac{1}{2}} \psi\left(\frac{x-b}{a}\right), \quad a, b \in \mathbb{R}, \quad a \neq 0 .
$$

Chebyshev wavelets $\psi_{n, m}(x)=\psi(k, n, m, x)$ have four arguments, $n=1,2, \cdots, 2^{k-1}, k$ is an arbitrary positive integer and $m$ is the order of Chebyshev polynomials of the first kind. They are defined on the interval $[0,1]$, as follows:

$$
\begin{aligned}
\psi_{n m}(x) & =\psi(k, n, m, x) \\
& =\left\{\begin{array}{lr}
2^{\frac{k}{2}} \tilde{T}_{m}\left(2^{k} x-2 n+1\right), & \frac{n-1}{2^{k-1}} \leq x<\frac{n}{2^{k-1}}, \\
0, & \text { otherwise }
\end{array}\right.
\end{aligned}
$$

where

$$
\tilde{T}_{m}(x)= \begin{cases}\frac{1}{\sqrt{\pi}}, & m=0, \\ \sqrt{\frac{2}{\pi}} T_{m}(x), & m>0 .\end{cases}
$$

For $m=0,1, \cdots, M-1$ and $n=1,2, \cdots, 2^{k-1}$.

$T_{m}(x), m=0,1, \cdots, M-1$, are the famous Chebyshev polynomials of the first kind of degree $m$, which construct an orthogonal system with respect to the weight function

$W(x)=\frac{1}{\sqrt{1-x^{2}}}$, on the interval $[-1,1]$, and satisfy the following recursive formula:

$$
\left\{\begin{array}{l}
T_{0}(x)=1, \\
T_{1}(x)=x, \\
T_{m+1}(x)=2 x T_{m}(x)-T_{m-1}(x), \quad m=1,2, \cdots
\end{array}\right.
$$

The set of Chebyshev wavelets is an orthogonal set with respect to the weight function $W_{n}(x)=W\left(2^{k} x-2 n+1\right)$.

A function $f(x)$ defined on the interval $[0,1]$ may be presented as

$$
f(x)=\sum_{n=1}^{\infty} \sum_{m=0}^{\infty} c_{n m} \psi_{n m}(x),
$$

where $c_{n m}=\left(f(x), \psi_{n m}(x)\right)_{W_{n}(x)}$. Series presentation

(7), can be approximated by the following truncated series.

$$
f(x) \simeq \sum_{n=1}^{2^{k-1}} \sum_{m=0}^{M-1} c_{n m} \psi_{n m}(x)=C^{T} \psi(x),
$$

where $C$ and $\psi(x)$ are $2^{k-1} M \times 1$ matrices given by

$$
\begin{aligned}
\boldsymbol{C} & \\
= & {\left[c_{10}, c_{11}, \ldots, c_{1 M-1}, c_{20}, c_{21}, \ldots, c_{2 M-1},\right.} \\
& \left.\quad \ldots, c_{2^{k-1} 0}, \ldots, c_{2^{k-1} M-1}\right]^{\mathrm{T}} \\
= & {\left[c_{1}, c_{2}, \ldots, c_{M}, c_{M+1}, \ldots, c_{2^{k-1} M}\right]^{\mathrm{T}}, }
\end{aligned}
$$

and

$$
\begin{aligned}
\psi & (x) \\
= & {\left[\psi_{10}(x), \psi_{11}(x), \cdots, \psi_{1, M-1}(x), \psi_{20}(x), \psi_{21}(x),\right.} \\
& \left.\cdots, \psi_{2, M-1}(x), \cdots, \psi_{2^{k-1} 0}(x), \cdots, \psi_{2^{k-1}, M-1}(x)\right]^{\mathrm{T}} \\
= & {\left[\psi_{1}(x), \psi_{2}(x), \cdots, \psi_{M}(x), \psi_{M+1}(x), \cdots, \psi_{2^{k-1} M}(x)\right]^{\mathrm{T}} . }
\end{aligned}
$$

Also a function $f(x, y)$ defined on $[0,1] \times[0,1]$ can be approximated as the following

$$
f(x, y) \simeq \psi^{T}(x) K \psi(y) .
$$

Here the entries of matrix $K=\left[k_{i j}\right]_{2^{k-1} M \times 2^{k-1} M}$ will be obtained by

$$
\begin{aligned}
k_{i, j}= & \left(\psi_{i}(x),\left(f(x, y), \psi_{j}(y)\right)_{W_{n}(y)}\right)_{W_{n}(x)}, \\
& i, j=1, \cdots, 2^{k-1} M .
\end{aligned}
$$

The integration of the vector $\psi(x)$, defined in (10), can be achieved as

$$
\int_{0}^{x} \psi(t) \mathrm{d} t=P \psi(x) .
$$

where $\boldsymbol{P}$ is the $2^{k-1} M \times 2^{k-1} M$ operational matrix of integration $[1,2]$. This matrix is determined as follows.

$$
\boldsymbol{P}=\left[\begin{array}{ccccc}
\boldsymbol{L} & \boldsymbol{F} & \boldsymbol{F} & \cdots & \boldsymbol{F} \\
\boldsymbol{O} & \boldsymbol{L} & \boldsymbol{F} & \ddots & \vdots \\
\boldsymbol{O} & \boldsymbol{O} & \boldsymbol{L} & \ddots & \boldsymbol{F} \\
\vdots & \ddots & \ddots & \ddots & \boldsymbol{F} \\
\boldsymbol{O} & \cdots & \boldsymbol{O} & \boldsymbol{O} & \boldsymbol{L}
\end{array}\right],
$$


where $\boldsymbol{F}, \boldsymbol{O}$ and $\boldsymbol{L}$ are $M \times M$ matrices given by

$$
\boldsymbol{F}=2^{-k}\left[\begin{array}{cccc}
2 & 0 & \cdots & 0 \\
0 & 0 & \cdots & 0 \\
-\frac{2 \sqrt{2}}{3} & 0 & \cdots & 0 \\
\vdots & \vdots & \ddots & \vdots \\
\frac{\sqrt{2}}{2}\left(\frac{1-(-1)^{r+1}}{r+1}-\frac{1-(-1)^{r-1}}{r-1}\right) & 0 & \cdots & 0 \\
\vdots & \vdots & \ddots & \vdots \\
\frac{\sqrt{2}}{2}\left(\frac{1-(-1)^{M}}{M}-\frac{1-(-1)^{M-2}}{M-2}\right) & 0 & \cdots & 0
\end{array}\right],
$$

$$
\boldsymbol{O}=\left[\begin{array}{cccc}
0 & 0 & \cdots & 0 \\
0 & 0 & \cdots & 0 \\
\vdots & \vdots & \ddots & \vdots \\
0 & 0 & \cdots & 0
\end{array}\right],
$$

The property of the product of two Chebyshev wavelets vector functions will be as follows

$$
\psi(x) \psi^{T}(x) Y \simeq \tilde{Y} \psi(x) .
$$

where $Y$ is a given vector and $\tilde{Y}$ is a $2^{k-1} M \times 2^{k-1} M$ matrix. This matrix is called the operational matrix of product. The integration of the product of two Chebyshev wavelets vector functions with respect to the weight function $W_{n}(x)$, is derived as

$$
\int_{0}^{1} W_{n}(x) \psi(x) \psi^{T}(x) \mathrm{d} x=\boldsymbol{I},
$$

where $\boldsymbol{I}$ is an identity matrix.

\section{Application of Chebyshev Wavelets Method}

In this section the introduced method will be applied to solve systems (1) and (2).

\subsection{Application to Solve System (1)}

Consider system of integro-differential Equations (1), with the following conditions

$$
u_{i}^{(s)}(0)=a_{i s}, \quad i=1, \cdots, n, s=0, \cdots, m-1 .
$$

First we assume the unknown functions $u_{i}^{(m)}(x), i=1,2, \cdots, n$ are approximated in the following forms

$$
u_{i}^{(m)}(x) \simeq C_{i}^{T} \psi(x), \quad i=1,2, \cdots, n .
$$

Therefore we have

$$
\begin{gathered}
u_{i}^{(s)}(x) \simeq C_{i}^{T} P^{m-s} \psi(x)+\sum_{j=0}^{m-1-s} a_{i s} \frac{x^{j}}{j !}, \\
i=1, \cdots, n, s=0, \cdots, m-1 .
\end{gathered}
$$

Using (20) and (21) other terms will be considered as the following general expansions

$$
\begin{aligned}
& f_{i}(x) \simeq F_{i}^{T} \psi(x), \\
& F_{i k}\left(x, u_{1}(x), u_{1}^{\prime}(x), \cdots, u_{1}^{(m)}(x), \cdots, u_{n}(x), \cdots, u_{n}^{(m)}(x)\right) \\
& \simeq X_{i k}^{T} \psi(x), \\
& G_{i j}\left(u_{1}(t), \cdots, u_{1}^{(m)}(t), \cdots, u_{n}(t), \cdots, u_{n}^{(m)}(t)\right) \simeq Y_{i j}^{T} \psi(t), \\
& k_{i j}(x, t) \simeq \psi^{T}(x) K_{i j} \psi(t), \\
& i=1,2, \cdots, n, k=1,2, \cdots, m_{1}, j=1,2, \cdots, m_{2} .
\end{aligned}
$$

$$
\boldsymbol{L}=2^{-k}\left[\begin{array}{ccccccc}
1 & \frac{1}{\sqrt{2}} & 0 & 0 & 0 & \cdots & 0 \\
-\frac{\sqrt{2}}{4} & 0 & \frac{1}{4} & 0 & 0 & \cdots & 0 \\
-\frac{\sqrt{2}}{3} & -\frac{1}{2} & 0 & \frac{1}{6} & 0 & \cdots & 0 \\
\vdots & \vdots & \vdots & \vdots & \ddots & \vdots & \vdots \\
\frac{\sqrt{2}}{2}\left(\frac{(-1)^{r-1}}{r-1}-\frac{(-1)^{r+1}}{r+1}\right) & 0 & 0 & \cdots & -\frac{1}{2(r-1)} & 0 & \frac{1}{2(r+1)} \\
\vdots & \vdots & \vdots & \vdots & \ddots & \vdots & \vdots \\
\frac{\sqrt{2}}{2}\left(\frac{(-1)^{M-2}}{M-2}-\frac{(-1)^{M}}{M}\right) & 0 & 0 & 0 & \cdots & -\frac{1}{2(M-2)} & 0
\end{array}\right],
$$


where $F_{i}$ and $K_{i j}$ are known matrices, $X_{i j}$ and $Y_{i j}$ are column vectors of elements of the vectors

$C_{i}, i=1, \cdots, n$.

Substituting these approximations into system (1), leads to

$$
\begin{aligned}
& C_{i}^{T} \psi(x)=F_{i}^{T} \psi(x)+\sum_{k=1}^{m_{1}} X_{i k}^{T} \psi(x) \\
& +\sum_{j=1}^{m_{2}} \int_{0}^{x} \psi^{T}(x) K_{i j} \psi(t) Y_{i j}^{T} \psi(t) \mathrm{d} t \\
& =F_{i}^{T} \psi(x)+\sum_{k=1}^{m_{1}} X_{i k}^{T} \psi(x) \\
& +\sum_{j=1}^{m_{2}} \psi^{T}(x) K_{i j}\left(\int_{0}^{x} \psi(t) Y_{i j}^{T} \psi(t) \mathrm{d} t\right) \\
& =F_{i}^{T} \psi(x)+\sum_{k=1}^{m_{1}} X_{i k}^{T} \psi(x)+\sum_{j=1}^{m_{2}} \psi{ }^{T}(x) K_{i j} \boldsymbol{T}_{i j} P \psi(x), \\
& \quad i=1,2, \cdots, n, k=1,2, \cdots, m_{1}, j=1,2, \cdots, m_{2} .
\end{aligned}
$$

where $\boldsymbol{T}_{i j}$ are $2^{k-1} M \times 2^{k-1} M$ matrices.

Multiplying $W_{n}(x) \psi^{T}(x)$, in to both sides of the system (23) and applying $\int_{0}^{1}(.) \mathrm{d} x$, a linear or a nonlinear system, in terms of the entries of $C_{i}, i=1,2, \cdots, n$, will be obtained and the elements of vectors $C_{i}, i=1,2, \cdots, n$ can be obtained by solving this system.

\subsection{Solving the System (2)}

Consider the system of integro-differential Equations (2) subject to the conditions (20). To solve this system, let's considered the unknown functions $u_{i}^{(m)}(x), i=1,2, \cdots, n$ as a linear combination of Chebyshev wavelets, by the following

$$
u_{i}^{(m)}(x) \simeq \psi^{T}(x) C_{i}, i=1,2, \cdots, n .
$$

and we have

$$
\begin{gathered}
u_{i}^{(s)}(x) \simeq \psi^{T}(x)\left(P^{T}\right)^{m-s} C_{i}+\sum_{j=0}^{m-1-s} a_{i s} \frac{x^{j}}{j !}, \\
i=1, \cdots, n, \quad s=0, \cdots, m-1
\end{gathered}
$$

Therefore the following general expansions are achieved.

$$
\begin{aligned}
& f_{i}(x) \simeq \psi^{T}(x) F_{i}, \\
& F_{i k}\left(x, u_{1}(x), u_{1}^{\prime}(x), \cdots, u_{1}^{(m)}(x), \cdots, u_{n}(x), \cdots, u_{n}^{(m)}(x)\right) \\
& \simeq \psi^{T}(x) X_{i k}, \\
& G_{i j}\left(u_{1}(t), \cdots, u_{1}^{(m)}(t), \cdots, u_{n}(t), \cdots, u_{n}^{(m)}(t)\right) \simeq \psi^{T}(x) Y_{i j}, \\
& k_{i j}(x, t) \simeq \psi^{T}(x) K_{i j} \psi(t), \\
& i=1,2, \cdots, n, k=1,2, \cdots, m_{1}, j=1,2, \cdots, m_{2} .
\end{aligned}
$$

Substitution of these approximations into the system (2), would be obtained results in

$$
\begin{aligned}
& \psi^{T}(x) C_{i}=\psi^{T}(x) F_{i}+\sum_{k=1}^{m_{1}} \psi^{T}(x) X_{i k} \\
& +\sum_{j=1}^{m_{2}} \int_{0}^{1} \psi^{T}(x) K_{i j} \psi(t) \psi^{T}(t) Y_{i j} \mathrm{~d} t \\
& =\psi^{T}(x) F_{i}+\psi^{T}(x) \sum_{k=1}^{m_{1}} X_{i k} \\
& +\psi^{T}(x) \sum_{j=1}^{m_{2}} K_{i j}\left(\int_{0}^{1} \psi(t) \psi^{T}(t) \mathrm{d} t\right) Y_{i j} \\
& =\psi^{T}(x) F_{i}+\psi^{T}(x) \sum_{k=1}^{m_{1}} X_{i k}+\psi^{T}(x) \sum_{j=1}^{m_{2}} K_{i j} D Y_{i j} \\
& \quad i=1,2, \ldots, n,
\end{aligned}
$$

where $\boldsymbol{D}$ is a $2^{k-1} M \times 2^{k-1} M$ matrix.

Multiplying both sides of the system (26) by $W_{n}(x) \psi(x)$ and applying $\int_{0}^{1}(.) \mathrm{d} x$, the following linear or non-linear system will be obtained

$$
C_{i}=F_{i}+\sum_{k=1}^{m_{1}} X_{i k}+\sum_{j=1}^{m_{2}} K_{i j} D Y_{i j}, i=1,2, \ldots, n,
$$

Solving system (27) the entries of $C_{i}, i=1,2, \cdots, n$, will be obtained.

Also one can check the accuracy of the method. Since the truncated Chebyshev wavelets series are approximate the solutions of the systems (1) and (2), so the error function $e\left(u_{i}(x)\right)$ is constructed as follows

$$
e\left(u_{i}(x)\right)=\left|u_{i}(x)-\sum_{n=1}^{2^{k-1}} \sum_{m=0}^{M-1} c_{n m}^{i} \psi_{n m}(x)\right| .
$$

If we set $x=x_{j}$ where $x_{j} \in[0,1]$, the error values can be obtained.

\section{Numerical Results}

In this section some systems of integro-differential equations are considered and solved by the introduced method. Parameters $k$ and $M$ are considered to be 1 and 6 respectively.

Example 1: Consider the following linear system of Fredholm integro-differential equations

$$
\left\{\begin{array}{l}
u^{\prime \prime}(x)+v^{\prime}(x)+\int_{0}^{1} 2 x t(u(t)-3 v(t)) \mathrm{d} t \\
=3 x^{2}+\frac{3}{10} x+8 \\
v^{\prime \prime}(x)+u^{\prime}(x)+\int_{0}^{1} 3\left(2 x+t^{2}\right)(u(t)-2 v(t)) \mathrm{d} t \\
=21 x+\frac{4}{5}, \quad 0 \leq x \leq 1 .
\end{array}\right.
$$


Subject to initial conditions

$u(0)=1, u^{\prime}(0)=0, v(0)=-1$ and $v^{\prime}(0)=2$. The exact solutions are $u(x)=3 x^{2}+1$ and $v(x)=x^{3}+2 x-1$ [8].

\section{Let's}

$$
\begin{aligned}
u^{\prime \prime}(x) & \simeq \psi^{T}(x) C_{1}, \quad v^{\prime \prime}(x) \simeq \psi^{T}(x) C_{2}, \\
u^{\prime}(x) & \simeq \psi^{T}(x) P^{T} C_{1}, \\
v^{\prime}(x) & \simeq \psi^{T}(x) P^{T} C_{2}+2=\psi^{T}(x) P^{T} C_{2}+\psi^{T}(x) V_{1}, \\
u(x) & \simeq \psi^{T}(x)\left(P^{T}\right)^{2} C_{1}+1 \\
& =\psi^{T}(x)\left(P^{T}\right)^{2} C_{1}+\psi^{T}(x) U_{0}, \\
v(x) & \simeq \psi^{T}(x)\left(P^{T}\right)^{2} C_{2}+2 x-1 \\
= & \psi^{T}(x)\left(P^{T}\right)^{2} C_{2}+\psi^{T}(x) V_{0}, \\
2 x t & \simeq \psi^{T}(x) K_{1} \psi(t), \quad 3\left(2 x+t^{2}\right) \simeq \psi^{T}(x) K_{2} \psi(t), \\
3 x^{2} & +\frac{3}{10} x+8 \simeq \psi^{T}(x) F_{1}, \quad 21 x+\frac{4}{5} \simeq \psi^{T}(x) F_{2},
\end{aligned}
$$

Substituting into (28), the following linear system will be obtained

$$
\left\{\begin{array}{l}
C_{1}+P^{T} C_{2}+V_{1} \\
\quad+K_{1}\left(D\left(P^{T}\right)^{2} C_{1}+U_{0}-3\left(P^{T}\right)^{2} C_{2}-3 V_{0}\right)=F_{1}, \\
P^{T} C_{1}+C_{2} \\
\quad+K_{2}\left(D\left(P^{T}\right)^{2} C_{1}+U_{0}-2\left(\left(P^{T}\right)^{2} C_{2}-2 V_{0}\right)=F_{2} .\right.
\end{array}\right.
$$

Solving this system; the solutions would be achieved as follows

$$
\begin{gathered}
u(x)=\psi^{T}(x)\left(P^{T}\right)^{2} C_{1}+1=3 x^{2}+1, \\
v(x)=\psi^{T}(x)\left(P^{T}\right)^{2} C_{2}+2 x-1=x^{3}+2 x-1 .
\end{gathered}
$$

This is the exact solution.

Example 2: Consider the following non-linear system of Fredholm integro-differential equations with the exact solutions $u(x)=x^{2}+1$ and $v(x)=\mathrm{e}^{x}$ and boundary conditions $u(0)=1, u^{\prime}(0)=0, v(0)=1$ and $v^{\prime}(0)=1$.

$$
\left\{\begin{array}{l}
u^{\prime \prime}(x)+4 v(x)+\int_{0}^{1}\left((x+t) u^{2}(t)+(2 x-t) v^{\prime}(t)\right) \mathrm{d} t \\
=4 \mathrm{e}^{x}+2 \mathrm{e} x-\frac{2}{15} x+\frac{13}{6} \\
v^{\prime \prime}(x)-3 u(x)+\frac{1}{2} \int_{0}^{1}\left((x-t) u^{\prime}(t) v^{\prime}(t)-(x+t) v^{3}(t)\right) \mathrm{d} t \\
=\mathrm{e}^{x}-3 x^{2}+\frac{7}{6} x-\frac{1}{6} \mathrm{e}^{3} x-\frac{1}{9} \mathrm{e}^{3}-\mathrm{e}-\frac{19}{18} \\
0 \leq x \leq 1
\end{array}\right.
$$

In this example, let's take

$$
\begin{aligned}
& u^{\prime \prime}(x) \simeq \psi^{T}(x) C_{1}, \\
& v^{\prime \prime}(x) \simeq \psi^{T}(x) C_{2}, \\
& u^{\prime}(x) \simeq \psi^{T}(x) P^{T} C_{1}, \\
& v^{\prime}(x) \simeq \psi^{T}(x) P^{T} C_{2}+1=\psi^{T}(x) P^{T} C_{2}+\psi^{T}(x) V_{1}, \\
& u(x) \simeq \psi^{T}(x)\left(P^{T}\right)^{2} C_{1}+1=\psi^{T}(x)\left(P^{T}\right)^{2} C_{1}+\psi^{T}(x) V_{1} \\
& v(x) \simeq \psi^{T}(x)\left(P^{T}\right)^{2} C_{2}+x+1 \\
& \quad \psi^{T}(x)\left(P^{T}\right)^{2} C_{2}+\psi^{T}(x) V_{0}, \\
& u^{2}(x) \simeq \psi^{T}(x) Y_{1}, \quad u^{\prime}(x) v^{\prime}(x) \simeq \psi^{T}(x) Y_{2}, \\
& v^{3}(x) \simeq \psi^{T}(x) Y_{3}, \\
& x+t \simeq \psi^{T}(x) K_{1} \psi(t), 2 x-t \simeq \psi^{T}(x) K_{2} \psi(t), \\
& x-t \simeq \psi^{T}(x) K_{3} \psi(t), \\
& 4 \mathrm{e}^{x}+2 \mathrm{e} x-\frac{2}{15} x+\frac{13}{6} \simeq \psi^{T}(x) F_{1}, \\
& \mathrm{e}^{x}-3 x^{2}+\frac{7}{6} x-\frac{1}{6} \mathrm{e}^{3} x-\frac{1}{9} \mathrm{e}^{3}-\mathrm{e}-\frac{19}{18} \simeq \psi^{T}(x) F_{2} .
\end{aligned}
$$

Applying the Chebyshev wavelets method, the following non-linear system will be obtained

$$
\left\{\begin{array}{l}
C_{1}+4\left(P^{T}\right)^{2} C_{2}+4 V_{0}+K_{1} D Y_{1}+K_{2} D\left(P^{T} C_{2}+V_{1}\right)=F_{1}, \\
C_{2}-3\left(P^{T}\right)^{2} C_{1}-3 V_{1}+\frac{1}{2} K_{3} D Y_{2}-\frac{1}{2} K_{1} D Y_{3}=F_{2} .
\end{array}\right.
$$

Solving this system the following results would be achieved.

$$
\begin{array}{ll}
c_{1,0}=2.506705578, & c_{1,1}=0.00009696248441, \\
c_{1,2}=0.00001788458767, & c_{1,3}=5.080260046 \times 10^{-7}, \\
c_{1,4}=-1.449170655 \times 10^{-7}, c_{1,5}=-2.373895017 \times 10^{-7}, \\
c_{2,0}=2.197451850, & c_{2,1}=0.7536322881, \\
c_{2,2}=0.09324678580, & c_{2,3}=0.007732766617, \\
c_{2,4}=0.0004818914245, & c_{2,5}=0.00002403741177 .
\end{array}
$$

Therefore, the following approximate solutions will be achieved.

$$
\begin{aligned}
u(x)= & 0.000001345778125 x^{5}+0.00001026370827 x^{4} \\
& +0.00001182646441 x^{3}+0.9999858747 x^{2} \\
& -1.336715265 \times 10^{-8} x+1 \\
v(x)= & 0.01391739110 x^{5}+0.03477166926 x^{4} \\
& +0.1704167644 x^{3}+0.4990585121 x^{2}+x+1 .
\end{aligned}
$$

Plots of the exact and approximate solutions are pre- 
sented in Figure 1 and also plots of error functions are shown in Figure 2.

Example 3: consider non-linear system of Volterra integro-differential equations with conditions

$u(0)=0, u^{\prime}(0)=1, u^{\prime \prime}(0)=0, v(0)=1, v^{\prime}(0)=0 \quad$ and $v^{\prime \prime}(0)=-1$.

$\left\{\begin{array}{l}u^{\prime \prime \prime}(x)=x-u^{\prime}(x)-\int_{0}^{x}\left(u^{\prime \prime 2}(t)+v^{\prime \prime 2}(t)\right) \mathrm{d} t, \\ v^{\prime \prime \prime}(x)=\sin x+\frac{1}{2} \sin ^{2} x+\int_{0}^{x} u^{\prime \prime}(t) v(t) \mathrm{d} t, 0 \leq x \leq 1 .\end{array}\right.$

The exact solutions are $u(x)=\sin x$ and $v(x)=\cos x \quad[7]$.

Entries of vectors $C_{1}$ and $C_{2}$ are computed by solving a system of nonlinear equations with six unknowns and six equations as follows

$$
\begin{array}{ll}
c_{1,0}=-1.032210259, & c_{1,1}=.2058700709, \\
c_{1,2}=0.04760379553, & c_{1,3}=-0.002178545560, \\
c_{1,4}=-0.0002500199685, & c_{1,5}=0.000006823287551, \\
c_{2,0}=0.5638994174, & c_{2,1}=0.3768424359, \\
c_{2,2}=-0.02600606157, & c_{2,3}=-0.003987821764, \\
c_{2,4}=0.0001365887993, & c_{2,5}=0.00001419890459 .
\end{array}
$$

Therefore, one gets the following approximate solutions

$$
\begin{aligned}
& u(x)=C_{1}^{T} P^{3} \psi(x)+x \\
&= 0.007222202515 x^{5}+0.001672032401 x^{4} \\
&-0.1676491115 x^{3}+0.0002483913114 x^{2} \\
&+x+0.3210839427 \times 10^{-7}, \\
& v(x)=C_{2}^{T} P^{3} \psi(x)-\frac{x^{2}}{2}+1 \\
&=-0.003945506187 x^{5}+0.04597489600 x^{4} \\
&-0.002189489246 x^{3}-\frac{1}{2} x^{2} \\
& \quad-0.00004265668610 x+1 .
\end{aligned}
$$

Approximate and exact solutions are plotted in Figure 3. Error functions are plotted in Figure 4.

Example 4: Consider the following non-linear system of Volterra integro-differential equations with the exact solutions $u(x)=x^{2}, \quad v(x)=x$ and $w(x)=3 x^{2}$ on $0 \leq x \leq 1 \quad[5]$.

$$
\left\{\begin{aligned}
u^{\prime \prime}(x)= & x+2 x^{3}+2 v^{\prime 2}(x)-\int_{0}^{x}\left(v^{\prime 2}(t)+u(t) w^{\prime \prime}(t)\right) \mathrm{d} t, \\
v^{\prime \prime}(x)= & -3 x^{2}-x u(t)+\int_{0}^{x}\left(x t v^{\prime}(t) u^{\prime \prime}(t)+w^{\prime}(t)\right) \mathrm{d} t, \\
w^{\prime \prime}(x)= & 2-\frac{4}{3} x^{3}+u^{\prime \prime 2}(t)-2 u^{2}(x) \\
& +\int_{0}^{x}\left(x^{2} v(t)+u^{\prime 2}(t)+t^{3} w^{\prime \prime}(t)\right) \mathrm{d} t,
\end{aligned}\right.
$$
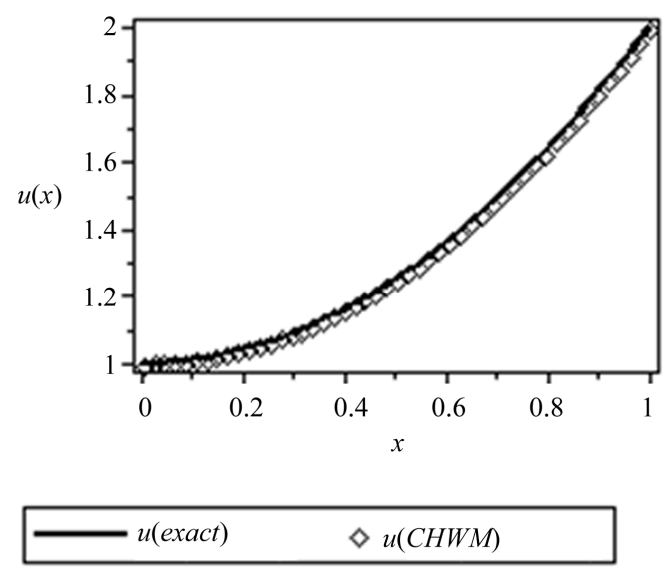

(a)
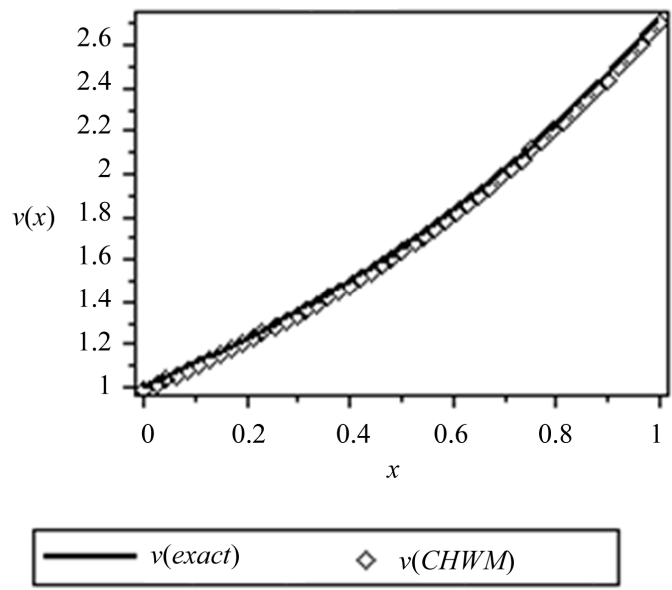

(b)

Figure 1. (a) and (b) plots of exact and approximate solutions of Example 2.

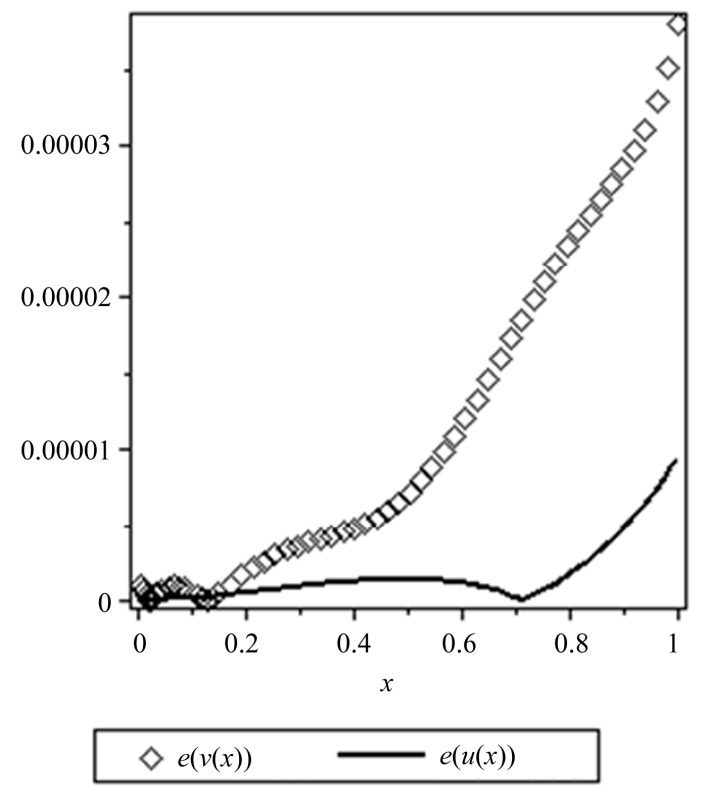

Figure 2. Plots of error functions of Examples 2. 

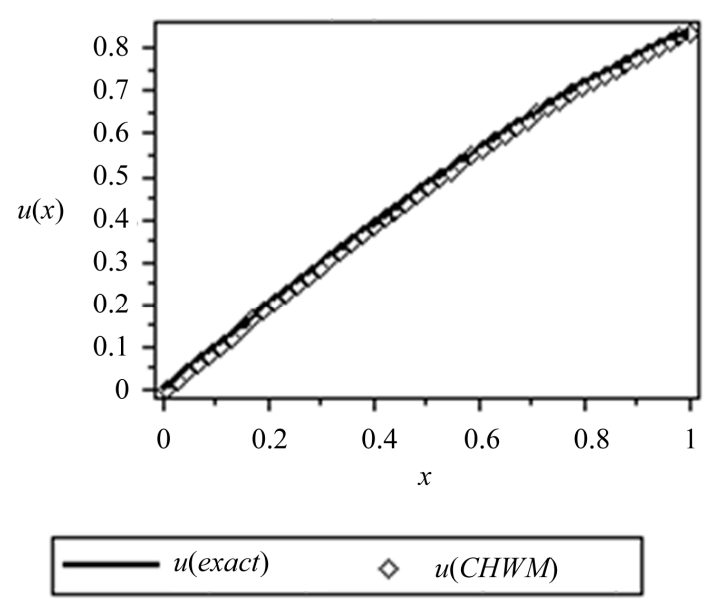

(a)
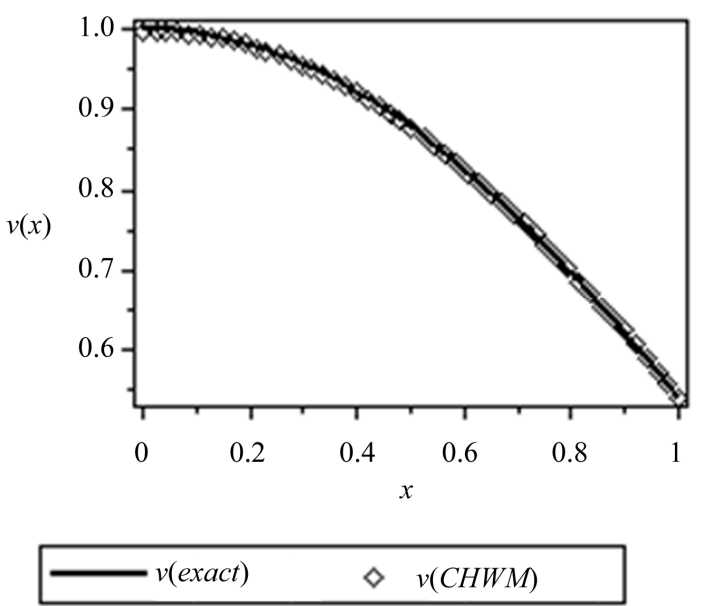

(b)

Figure 3. (a) and (b) plots of exact and approximate solutions of Example 3.

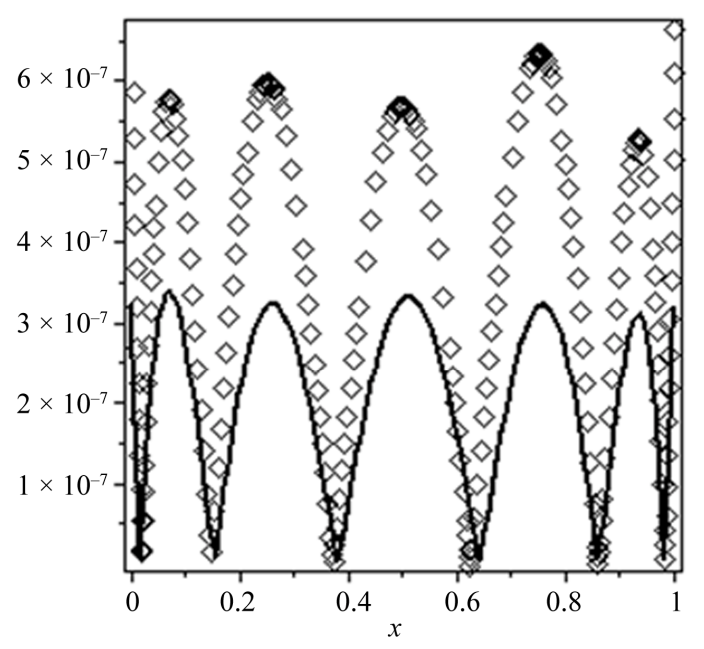

$$
\diamond e(v(x)) \quad \longrightarrow e(u(x))
$$

Figure 4. Plots of error functions of Examples 3.
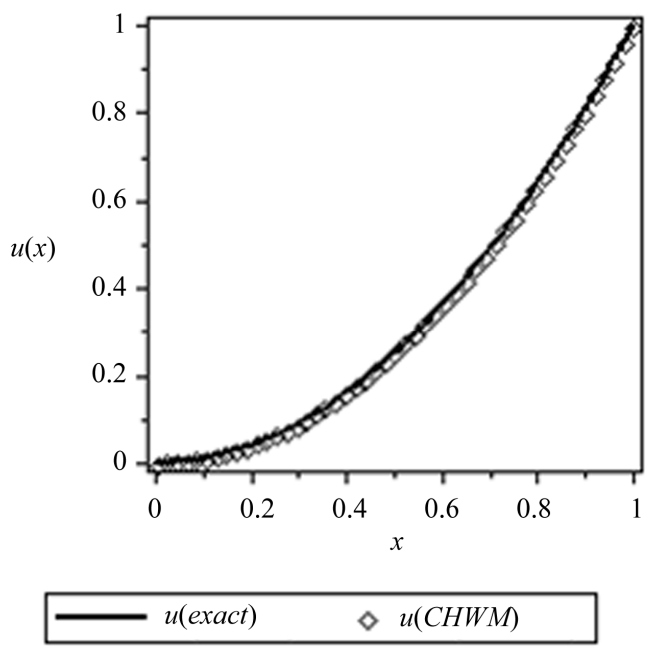

(a)
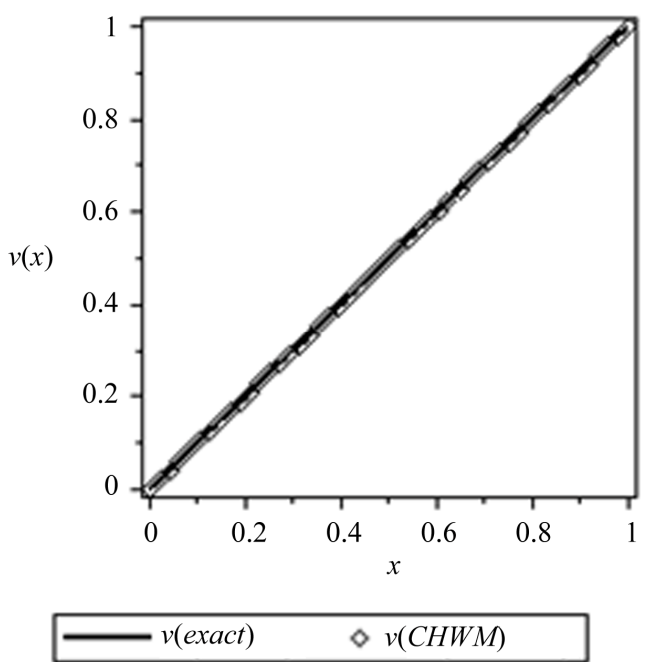

(b)

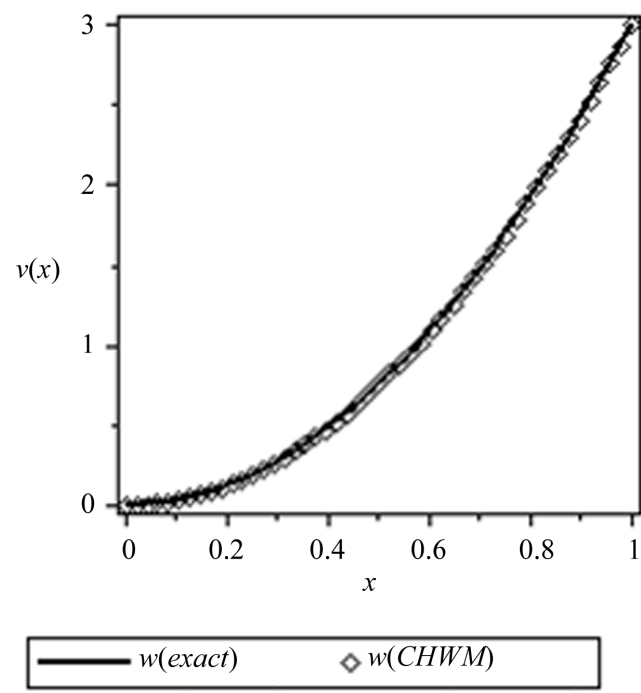

(c)

Figure 5. (a), (b) and (c) plots of exact and approximate solutions of Example 4. 


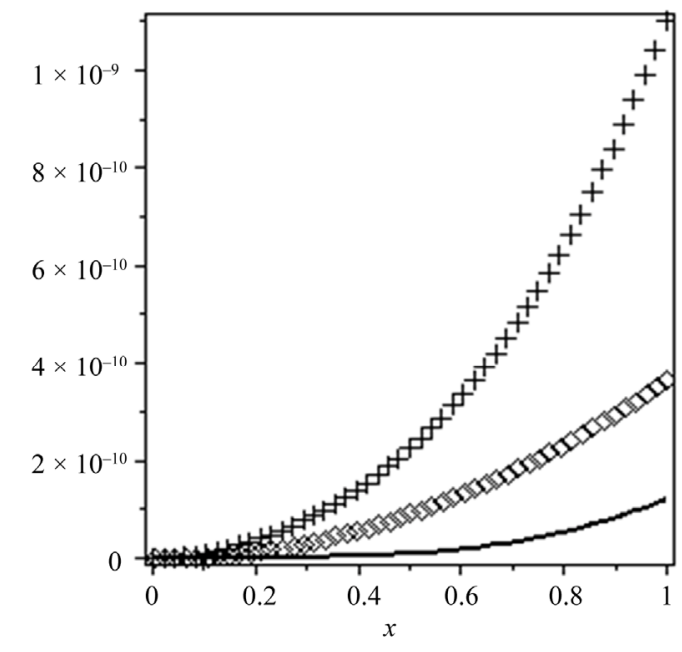

$\diamond e(v(x)) \quad \longrightarrow e(u(x))+e(w(x))$

Figure 6. Plots of error functions of Examples 4.

Initial conditions are $u(0)=0, u^{\prime}(0)=0$, $v(0)=0, v^{\prime}(0)=1, \quad w(0)=0 \quad$ and $w^{\prime}(0)=0$.

The following approximate solutions would be achieved by using Chebyshev wavelets method.

$$
\begin{aligned}
u(x)=C_{1}^{T} P^{2} \psi(x) \\
=-8.527181902 \times 10^{-11} x^{5}+2.509321144 \times 10^{-10} x^{4} \\
-4.653979568 \times 10^{-11} x^{3}+x^{2}+3.641136902 \times 10^{-13} x \\
-6.551942965 \times 10^{-15}, \\
v(x)=C_{2}^{T} P^{2} \psi(x)+x \\
=7.880203686 \times 10^{-11} x^{5}-1.637978948 \times 10^{-10} x^{4} \\
\quad+1.014393404 \times 10^{-10} x^{3}+3.511874249 \times 10^{-10} x^{2} \\
\quad+x-1.461792049 \times 10^{-14}, \\
w(x)=C_{3}^{T} P^{2} \psi(x) \\
=-4.043702026 \times 10^{-10} x^{5}+1.228923763 \times 10^{-9} x^{4} \\
\quad-7.249989732 \times 10^{-10} x^{3}+3.000000001 x^{2} \\
\quad+1.753892960 \times 10^{-12} x-2.955310652 \times 10^{-14} .
\end{aligned}
$$

Plots of the exact solution, approximate solution and error functions are shown in Figures 5 and 6.

\section{Conclusions}

This paper proposes a powerful technique for solving systems of integro-differential equations using Chebyshev wavelets method. Comparison of the approximate solutions and the exact solutions shows that the proposed method is more efficient tool and more practical for solving linear and non-linear systems of integro-difierential equations, and plots confirm. Researches for finding more applications of this method and other orthogonal basis functions are one of the goals in our research group. The package Maple 13 has been used to carry the computations associated with these examples.

\section{Acknowledgements}

Authors are grateful to the anonymous reviewers for his (her) influential comments which have improved the quality of the paper.

\section{References}

[1] E. Babolian and F. Fattahzadeh, "Numerical Computation Method in Solving Integral Equations by Using Chebyshev Wavelet Operational Matrix of Integration," Applied Mathematics and Computations, Vol. 188, No. 1, 2007, pp. 1016-1022. doi:10.1016/j.amc.2006.10.073

[2] E. Babolian and F. Fattahzadeh, "Numerical Solution of Differential Equations by Using Chebyshev Wavelet Operational Matrix of Integration," Applied Mathematics and Computations, Vol. 188, No. 1, 2007, pp. 417-426.

[3] Y. Li, "Solving a Nonlinear Fractional Differential Equation Using Chebyshev Wavelets," Communications in Nonlinear Science and Numerical Simulation, Vol. 15, No. 9, 2010, pp. 2284-2292. doi:10.1016/j.cnsns.2009.09.020

[4] J. Biazar, "Solution of Systems of Integral-Differential Equations by Adomian Decomposition Method," Applied Mathematics and Computation, Vol. 168, No. 2, 2005, pp. 1232-1238. doi:10.1016/j.amc.2004.10.015

[5] J. Biazar, H. Ghazvini and M. Eslami, "He's Homotopy Perturbation Method for Systems of Integro-Differential Equations," Chaos, Solitions and Fractals, Vol. 39, No. 3, 2009, pp. 1253-1258. doi:10.1016/j.chaos.2007.06.001

[6] E. Yusufoglu (Agadjanov), "An Efficient Algorithm for Solving Integro-Differential Equations System," Applied Mathematics and Computation, Vol. 192, No. 1, 2007, pp. 51-55. doi:10.1016/j.amc.2007.02.134

[7] J. Biazar and H. Aminikhah, "A New Technique for Solving Integro-Differential Equations," Computers and Mathematics with Applications, Vol. 58, No. 11-12, 2009, pp. 2084-2090. doi:10.1016/j.camwa.2009.03.042

[8] J. Pour-Mahmoud and M. Y. Rahimi-Ardabili and S. Shamorad, "Numerical Solution of the System of Fredholm Integro-Differential Equations by the Tau Method," Applied Mathematics and Computation, Vol. 168, 2005, pp. 465-478.

[9] S. Abbasbandy and A. Taati, "Numerical Solution of the System of Nonlinear Volterra Integro-Differential Equations with Nonlinear Differential Part by the Operational Tau Method and Error Estimation," Journal of Computational and Applied Mathematics, Vol. 231, No. 1, 2009, pp. 106-113. doi:10.1016/j.cam.2009.02.014

[10] A. Arikoglu and I. Ozkol, "Solutions of Integral and Integro-Differential Equation Systems by Using Differential Transform Method," Computers and Mathematics 
with Applications, Vol. 56, No. 9, 2008, pp. 2411-2417. doi:10.1016/j.camwa.2008.05.017

[11] M. Gachpazan, "Numerical Scheme to Solve IntegroDifierential Equations System," Journal of Advanced Research in Scientific Computing, Vol. 1, No. 1, 2009, pp. 11-21.

[12] K. Maleknejad, F. Mirzaee and S. Abbasbandy, "Solving Linear Integro-Differential Equations System by Using Rationalized Haar Functions Method," Applied Mathematics and Computation, Vol. 155, No. 2, 2004, pp. 317328. doi:10.1016/S0096-3003(03)00778-1
[13] K. Maleknejad and M. Tavassoli Kajani, "Solving Linear Integro-Differential Equation System by Galerkin Methods with Hybrid Functions," Applied Mathematics and Computation, Vol. 159, No. 3, 2004, pp. 603-612. doi:10.1016/j.amc.2003.10.046

[14] I. Daubeches, "Ten Lectures on Wavelets," SIAM, Philadelphia, 1992.

[15] Ole Christensen and K. L. Christensen, "Approximation Theory: From Taylor Polynomial to Wavelets," Birkhauser, Boston, 2004. 